\title{
Impact of jaw position on sparing organs at risk in 3-dimensional conformal radiation therapy of pancreatic cancer
}

\author{
Nava Paudel1, Ganesh Narayanasamy', Eun-Young Han², Xin Zhang1, Bishnu Thapa ${ }^{3}$, \\ Jose Penagaricano1, Xiaoying Liang1, Steven Morrill ${ }^{1}$ \\ 1Department of Radiation Oncology, University of Arkansas for Medical Sciences, Little Rock, Arkansas, USA \\ 2 Department of Radiation Physics, UT MD Anderson Cancer Center, Houston, Texas, USA \\ ${ }^{3}$ Department Radiation Oncology, Tallahassee Memorial Cancer Center, Tallahassee, Florida, USA
}

Received November 10, 2015; Revised March 02, 2016; Accepted March 04, 2016; Published Online March 07, 2016

\section{Technical Report}

\begin{abstract}
Purpose: The objective of this work is to investigate the impact of collimator jaw position on dose to organs at risk (OARs) during a 3-dimensional conformal radiotherapy (3DCRT) of pancreatic cancer and postulate a method to minimize OAR dose by proper positioning of the jaws. Methods: Clinically delivered 3DCRT treatment plans for 10 patients optimized with multiple static beams using multileaf collimator (MLC) leaves conformed to a block margin around target, and collimator jaws aligned with outer extent of the block margin were selected. Subsequent plans were generated by displacing the collimator jaws outward in lateral, superior-inferior or both directions by 1 and $2 \mathrm{~cm}$ without altering the MLC position. Computed dose to OARs and target with unaltered dose normalization were compared against the corresponding dose obtained from the original plans. Results: Outward displacement of the collimator jaws by $1 \mathrm{~cm}$ in lateral and/or superior-inferior direction resulted in a significant increase in mean dose to the studied OARs. The increase was found to be proportional to the outward displacement of the jaws. The increase in maximum dose to spinal cord was significant in a few patients while it was insignificant for all other OARs. Conclusion: Collimator jaws aligned with outer extent of a block margin minimize dose to OARs. Any gap between the block margin and the collimator jaws can lead to an inadvertent delivery of higher dose to the OARs. Hence, the use of an optimal jaw position during treatment planning becomes important to all patient plans.
\end{abstract}

Keywords: 3D Conformal Radiotherapy; Pancreatic Cancer; Collimator Jaws; OARs; HD MLCs; Varian TrueBeam Linear Accelerator; Enhanced Dynamic Wedge (EDW)

\section{Introduction}

Pancreatic cancer has been projected to become second leading cause of cancer related death in the United States by $2030 .^{1}$ Surgery alone is not an obvious option for pancreatic cancer treatment because of its aggressive biology, late diagnosis, encasement of large blood vessels and the presence of metastasis. ${ }^{2,3}$ Despite the high chances of distant metastases of pancreatic cancer, radiotherapy may provide a survival advantage. ${ }^{4}$ Current radiation prescription dose ( $\sim 54$ Gy) is not adequate for the tumor control.5,6 One approach for better tumor control is to increase the prescription dose but that option comes at the cost of higher toxicity to the OARs. ${ }^{7}$ Hence reducing dose to OARs becomes extremely important.

Intensity modulated radiation therapy (IMRT) is a technique that can spare critical structures well. But some studies suggest that IMRT did not present a significant advantage over three dimensional conformal radiation therapy (3DCRT) in terms of OARs sparing. ${ }^{8}$ 
There are also potential problems of low dose spread over larger volume and higher leakage dose due to the usage of higher monitor units (MUs) during an IMRT treatment. Nonetheless, selection of radiotherapy treatment modality depends on the staging of tumor and confidence level of a physician among other factors; and 3DCRT remains a widely used modality for treating pancreatic cancer.

Usually, tumors have a complex shape and collimator jaws of a linear accelerator (LINAC) alone cannot conform to the targets. Multileaf collimators (MLCs) incorporated in modern LINACs offer a good conformity with the target but leaf transmission leads to a higher radiation dose delivery as compared to the collimator jaws. Rounded end MLCs suffer from bigger transmission penumbra and higher leaf-end radiation transmission. ${ }^{9}$

For the current standard prescription dose to the target, 3DCRT plans usually meet the clinical standards. However, optimal jaw position can help reduce dose to OARs surrounding the target. ${ }^{10}$ Dose reduction to OARs can help escalate the prescription dose, or at least reduce the chances of complication to the OARs. Minimum Y-jaw separation required for enhanced dynamic wedge (EDW) in some LINACs may not allow for the use of an optimal jaw position in some of the 3DCRT plans. This factor as well as a gap introduced between the jaws and a block margin during treatment planning can increase dose to the surrounding critical structures. Here we perform a quantitative assessment of the effect of jaw position on dose to OARs and present a method to minimize dose to the OARs during a 3DCRT treatment of pancreatic cancer.

\section{Methods and Materials}

\subsection{Patient population}

Ten pancreatic cancer patients treated with 3DCRT were selected in random for the study. These patients were imaged with a computed tomography (CT) simulator (Phillips Brilliance Big Bore, Amsterdam, Netherlands) for treatment planning. Treatment plans were generated with Eclipse treatment planning system (Version 11.0.47, Varian Medical Systems, Palo Alto, CA) using the analytical anisotropic algorithm (AAA). The patients were treated to 45-50.4 Gy (1.8 Gy/fraction) using 18 MV photon beam from a Varian TrueBeam STx LINAC with rounded leaf end 120 high-definition MLCs. OARs (left and right kidneys, stomach, liver, cord and bowel) and target (planning target volume- PTV) were contoured.

PTV volume among the patients ranged from 56 to 896 cc. While uniform $7 \mathrm{~mm}$ margin around PTV was enough for intended target coverage in 8 patients, $8 \mathrm{~mm}$ was needed in 2 cases. Four to seven static beams (at four cardinal angles, plus oblique gantry angles for additional beams with collimator angles of $0^{0}$ or $90^{\circ}$ ) were used to generate each treatment plan. The beam angles and EDW (a virtual wedge created by a computer controlled Y-jaw movement and dose rate change in Varian LINACs) were used as needed in order to get good target coverage while minimizing dose to the OARs. Each treatment plan was normalized to cover 95\% of PTV volume by $100 \%$ of the prescribed dose with the collimator jaws aligned with outer extent of the block outline as shown in Figure 1(a). Dose to PTV and OARs were evaluated with isodose distributions and dose volume histograms (DVHs).

\subsection{Research plans}

Starting from the clinical treatment plan, subsequent research plans were generated by translating two opposite jaws away from the treatment field. The jaws were moved in superior-inferior (refer Figure 1(b)), lateral or both directions by $1 \mathrm{~cm}$ and $2 \mathrm{~cm}$. Note that the MLC positions were not altered in those plans. Dose to the target and OARs was calculated for every research plan keeping the same PTV dose normalization. Preserved dose normalization did not alter MUs used in any of the treatment plans.

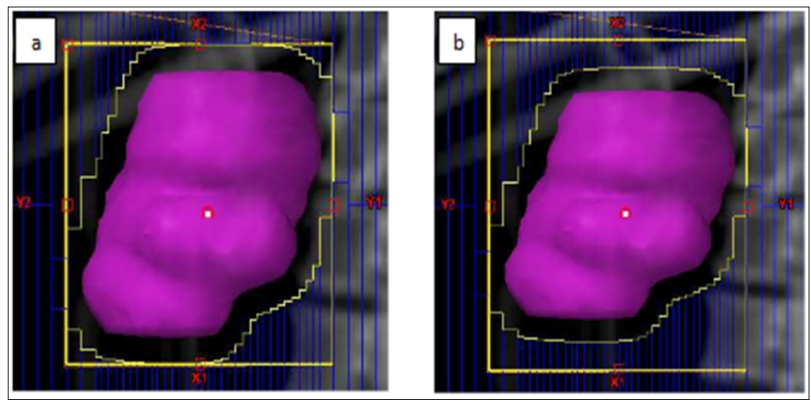

Figure 1: Collimator jaws (a) Aligned with the outer extent of a block margin around PTV in a clinical plan; (b) Displaced by $1 \mathrm{~cm}$ in superior inferior direction.

Mean and maximum dose to the OARs for various jaw positions were obtained from the treatment planning system and compared against the corresponding dose obtained from the delivered clinical plans. As there were differences in mean and maximum dose to the OARs in the research plans in comparison with the clinical plans, the significance of the differences was evaluated using statistical analysis.

\subsection{Statistical analysis}

Test for normal distribution was performed using Shapiro-Wilk test in the $\mathrm{R}$ statistical package. ${ }^{\mathbf{1 1}}$ Statistical significance was tested for normally distributed data using a paired Student's T-test. For a distribution showing larger deviation from a normal distribution, a Wilcoxon signed-rank test was used. Statistical significance was compared against a threshold $p$-value of 0.05 . 


\section{Results}

Range of the change in mean dose to target and various OARs due to lateral or superior-inferior displacement of jaws by $1 \mathrm{~cm}$ are tabulated in Table 1 . Changes in mean dose to the OARs were larger than the changes in maximum dose. The changes in dose were small and not so apparent in DVHs for the $1 \mathrm{~cm}$ jaw displacement.

As evident from Table 1, mean dose to most of the OARs increased approximately by $1 \%$ due to $1 \mathrm{~cm}$ outer displacement of the jaws. This increase was found up to $5 \%$ in some cases. Student t-test and Wilcoxon signed rank test showed that the mean dose differences are significant $(p<0.02)$. Kidneys, stomach and cord dose were most affected by an increased jaw margin. These effects on most of the patients were smaller for larger structures such as liver and bowels, and other structures that extend beyond $2 \mathrm{~cm}$ from the target. However, the increase was up to $3.2 \%$ for liver in a patient. There was insignificant change in mean dose to the PTV, as expected from the preservation of plan normalization.

Table 1: Range of percentage increase in mean dose (\% $\Delta \mathrm{D}_{\text {mean }}$ ) to PTV and OARs due to $1 \mathrm{~cm}$ jaw displacement along the lateral or superior-inferior direction for 10 patients.

\begin{tabular}{lcc}
\hline \hline Structure & $\% \Delta \mathrm{D}_{\text {mean }}$ (Lateral) & $\% \Delta \mathrm{D}_{\text {mean }}$ (Sup-inf) \\
\hline Lt. kidney & $0.4-2.0$ & $-0.2-0.8$ \\
Rt. kidney & $0.4-2.9$ & $-0.1-2.0$ \\
Bilat. kidney & $0.3-2.1$ & $-0.1-0.9$ \\
Cord & $0.0-2.6$ & $0.2-1.1$ \\
Stomach & $0.2-1.3$ & $0.5-5.3$ \\
Bowel & $0.1-1.1$ & $0.2-1.5$ \\
Liver & $0.3-1.2$ & $-0.0-3.2$ \\
PTV & $0.0-0.1$ & -0.1 \\
\hline \hline
\end{tabular}

The increase in mean dose from $1 \mathrm{~cm}$ and $2 \mathrm{~cm}$ lateral and superior-inferior displacement of jaws averaged over 10 patients are shown in Figure 2 and Figure 3 respectively.

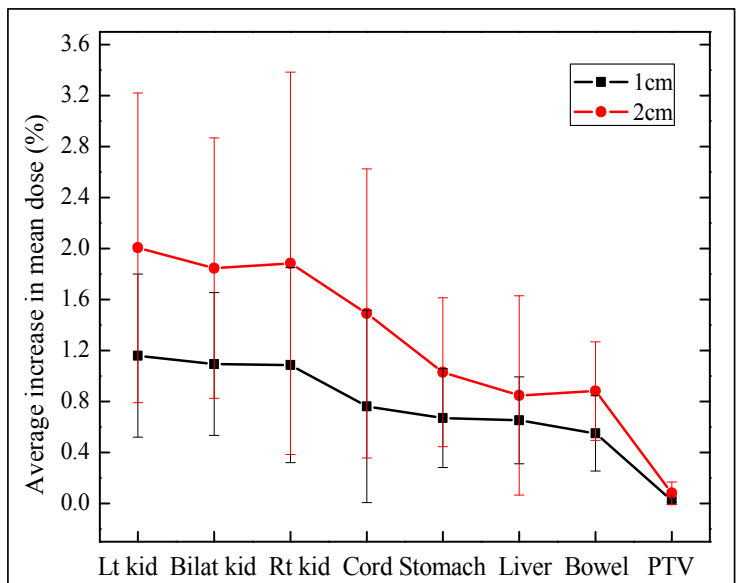

Figure 2: Effect of outward (lateral) displacement of jaws on mean dose to OARs and target.

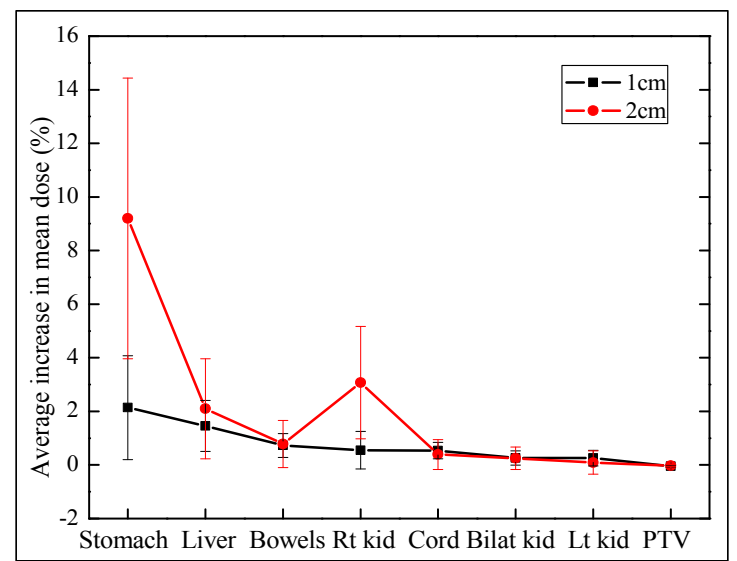

Figure 3: Effect of outward (superior-inferior) displacement of jaws on mean dose to OARs and target.

Results from Figure 2 and 3 show that structures lying laterally outwards from the target (kidneys, and liver) in most patients were less affected by the superior-inferior displacement of the jaws. An exception was the liver where higher increase in dose was observed in a patient. Likewise, liver and bowel were less affected by lateral jaw displacement. As evident from Figure 2, the percentage increase in mean dose to the OARs increased linearly with the increase in outward lateral displacement of the jaws. Even though changes in maximum dose to most of the OARs were small, meaningful changes were observed for cord in few patients. Maximum dose, a limiting parameter for the cord, increased up to $3 \%(\sim 100 \mathrm{cGy})$ for $1 \mathrm{~cm}$ superior-inferior displacement of the jaws and increased slightly with the increase in jaw displacement.

Outward displacement of all four jaws resulted in a much higher increase in mean dose to the OARs than only with one jaw pair displacement. Such increase was higher for larger displacement of both jaw pairs. Effect of $2 \mathrm{~cm}$ jaw displacement on DVHs for a few critical structures of a representative patient is shown in Figure 4.

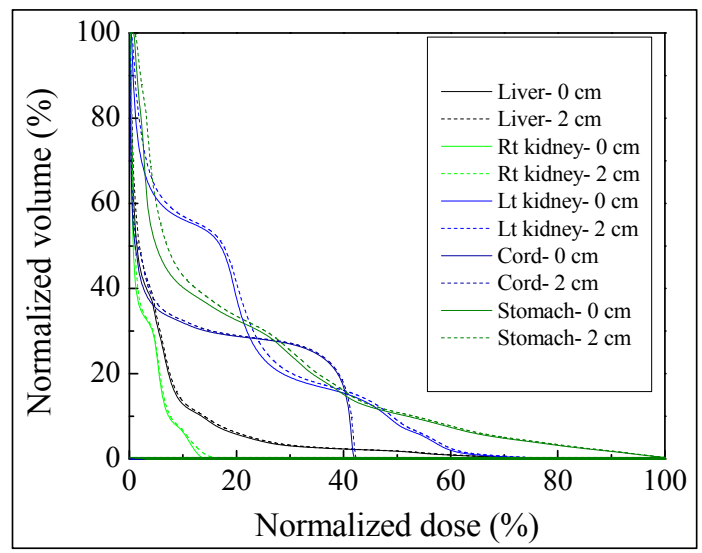

Figure 4: Comparison of the effect of outward displacement of all 4 jaws by $2 \mathrm{~cm}$ against no (zero) displacement (dose is normalized with respect to the highest dose among the OARs). 


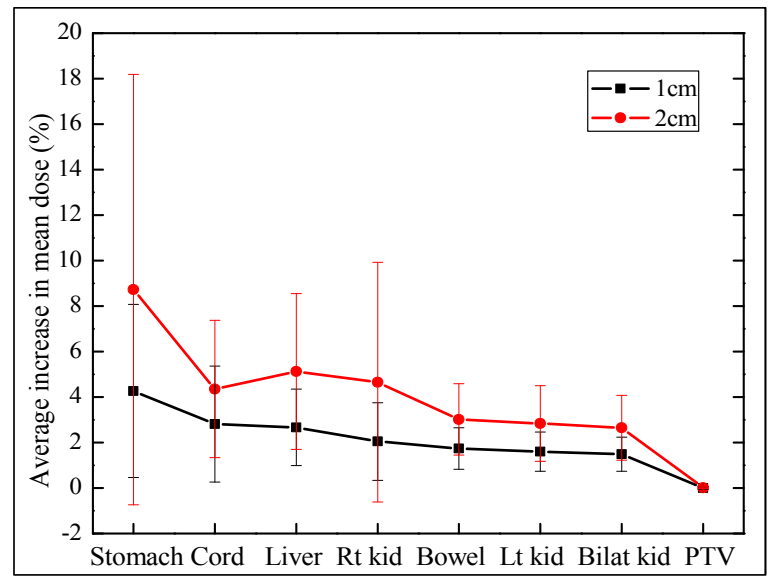

Figure 5: Effect of outward displacement of all four jaws by 1 and $2 \mathrm{~cm}$ on mean dose to OARs.

Increase in mean dose to various structures averaged over 10 patients is presented in Figure 5. As evident from Figure 5, increase in mean dose to OARs averaged over 10 patients ranged from 1.5 to $4.3 \%$ for $1 \mathrm{~cm}$ outward displacement of all 4 jaws and the values almost doubled for $2 \mathrm{~cm}$ displacement. But the increase was $0.0 \%$ for PTV in both cases. Among the patients studied, the increase in mean dose to stomach for $1 \mathrm{~cm}$ jaw displacement was as high as $144 \mathrm{cGy}$. The increase in mean dose to other structures in some patients ranged as high as 50-100 cGy. Such increases are statistically significant and can have clinical significance in some structures. The increase in mean dose to some of the OARs in few patients were much larger than the averaged values displayed in the figures. Since such a large increase may occur in any patient, a careful consideration should be given to minimize it.

\section{Discussion}

Our study did not show any obvious correlation between the percentage increase in mean dose to the OARs and any other clinical parameters such as PTV size. It is obvious that PTV shape and location differ with patients. Hence portion of the critical structures lying underneath the gap between the inner extent of MLCs and jaws varied with complexity of the PTV shape. This variation in gap resulted in a different amount of increase in mean dose to the OARs. The increase in absolute dose to the OARs increases with the increase in MUs for the same field size provided the area between MLC leaf end and jaw position is constant. It also depends on the number of fields and size of the field. However, the area between MLC ends and jaws changes with complexity of the target shape resulting in a varying transmission. Treatment parameters including the field size, number of fields and the number of MUs depend on the size, shape and location of the target, beam modulation and prescription dose, leaving the treatment planner with less choice. However, area between MLC leaves and jaw position can always be minimized to reduce dose to the OARs.
Generally, MLCs conform to the outer extent of a block margin and a few millimeter gap between inner extent of MLCs and the collimator jaws is typical in a 3DCRT plan. However, there can be a different scenario in some cases. As an example, if a planner decides to reduce the margin around the PTV, MLCs move to conform to the new block margin leaving the jaws in their original position. Some treatment planning systems such as Eclipse do not allow a merger of fields with different jaw defined field sizes. Hence the usage of subfields in a field does not reduce the gap between a block margin and the jaws and the leakage dose around the treatment target does not decrease. In addition, the use of an EDW in Varian machines requires a minimum of $4 \mathrm{~cm}$ Y-jaw separation. Hence, an EDW might not permit the optimal jaw position for small target treatment. These effects should be carefully considered during treatment planning. Without a careful consideration, OARs underneath the MLCs can receive an unnecessarily higher dose.

The role of an optimal jaw position in reducing dose to normal structure in 3DCRT based intracranial stereotactic radiosurgery (SRS) planning has been investigated and our results are in line with the results from Han et al. ${ }^{12}$

In spite of a low dose spill outside the treatment area, IMRT and volumetric modulated arc therapy (VMAT) plans offer a good OAR sparing. VMAT provides a faster dose delivery with OAR sparing comparable to IMRT. 13,14 Comparative studies on pancreatic cancer treatment show that VMAT and IMRT plans provide a better dose conformity than 3DCRT plans. In addition, VMAT produces a dose conformity comparable to IMRT while reducing the dose delivery time the and the number of MUs used. 15,16

\section{Conclusion}

Collimator jaws aligned with the outer extent of a block margin offer the optimal position in minimizing dose to OARs during a 3DCRT delivery. Any outward displacement of the jaws from the optimal position can increase dose to OARs surrounding the treatment target. This effect is patient specific and depends on the gap between the inner extents of MLCs and the jaws, and target shape and location relative to the OARs. Even a small outward displacement of the jaws may lead to a substantial increase in OARs dose that may have a clinical significance.

Based on the study, we highly recommend that the collimator jaws be pulled all the way in to match with the block outline around the target in all external beam plans including 3DCRT. Reduction in dose to normal structures not only lowers the chances of normal tissue complication including the risk of secondary cancer, it also helps keep the door open for prescription radiation 
dose escalation or for a boost treatment for the better tumor control.

\section{Conflict of interest}

The authors declare that they have no conflicts of interest. The authors alone are responsible for the content and writing of the paper.

\section{References}

1. Pancreatic cancer projected to become second leading cause of cancer-related death in the United States by 2030; AACR News release, 2015 available from http://www.aacr.org/Newsroom/Pages/News-Re lease-Detail.aspx?ItemID=541\#.Ve2ZYhFVhBc

2. Ghaneh P, Costello E, Neoptolemos JP. Biology and management of pancreatic cancer. Gut. 2007; 56: 1134-52.

3. Tempero MA, Arnoletti JP, Behrman S, et al. Pancreatic adenocarcinoma. J Natl Compr Canc Netw. 2010; 8(9): 972-1017.

4. Treatment of locally unresectable carcinoma of the pancreas: comparison of combined-modality therapy (chemotherapy plus radiotherapy) to chemotherapy alone. Gastrointestinal Tumor Study Group. J Natl Cancer Inst. 1988;80(10):751-5.

5. Roldan GE, Gunderson LL, Nagorney DM, et al. External beam versus intraoperative and external beam irradiation for locally advanced pancreatic cancer. Cancer. 1988; 61: 1110-6.

6. Klaassen DJ, MacIntyre JM, Catton GE, et al. Treatment of locally unresectable cancer of the stomach and pancreas: a randomized comparison of 5-fluorouracil alone with radiation plus concurrent and maintenance 5-fluorouracil--an Eastern Cooperative Oncology Group study. J Clin Oncol. 1985; 3: 373-8.

7. Ceha HM, van Tienhoven G, Gouma DJ, et al. Feasibility and efficacy of high dose conformal radiotherapy for patients with locally advanced pancreatic carcinoma. Cancer. 2000; 89 2222-9.

8. Ling TC, Slater JM, Mifflin R, et al. Evaluation of normal tissue exposure in patients receiving radiotherapy for pancreatic cancer based on RTOG 0848. J Gastrointest Oncol. 2015; 6(2): 108-14.

9. Szpala S, Cao F, Kohli K. On using the dosimetric leaf gap to model the rounded leaf ends in VMAT/RapidArc plans. J Appl Clin Med Phys. 2014;15(2):4484.

10. Paudel N, Han E, Liang X, et al. Effect of jaw position on dose to critical structures in 3-D conformal radiotherapy treatment of pancreatic cancer. Med Phys. 2015;42(6):3413.

11. R Development Core Team. R: A language and environment for statistical computing. $\mathrm{R}$ Foundation for Statistical Computing, Vienna, Austria. ISBN 3-900051-07-0, 2008.

12. Han EY, Zhang X, Yah Y, et al. Static jaw collimation settings to minimize radiation dose to normal brain tissue during stereotactic radiosurgery. Med Dosim. 2012;37:391-5.

13. Ali MA, Babaiah M, Madhusudhan N, et al. Comparative dosimetric analysis of IMRT and VMAT (RapidArc) in brain, head and neck, breast and prostate malignancies. Int J Cancer Ther Oncol. 2015;3(1):03019.

14. Amoush A, Dalton A, Rabatic B, et al. Volumetric modulated arc therapy for spine SBRT patients to reduce treatment time and intrafractional motion. Int J Cancer Ther Oncol. 2015; 3(2):03026.

15. Eppinga W, Lagerwaard F, Verbakel W, et al. Volumetric modulated arc therapy for advanced pancreatic cancer. Strahlenther Onkol. 2010;186(7):382-7.

16. Nabavizadeh N, Simeonova AO, Waller JG, et al. Volumetric-modulated arc radiotherapy for pancreatic malignancies: dosimetric comparison with sliding-window intensity -modulated radiotherapy and 3-dimensional conformal radiotherapy. Med Dosim. 2014; 39(3):256-60. 